\title{
Design and experimental testing of an adaptive shape-morphing tensegrity structure, with frequency self-tuning capabilities, using shape-memory alloys
}

\author{
Filipe Amarante dos $\operatorname{Santos}^{1}$, André Rodrigues ${ }^{1}$, \\ Andrea Micheletti ${ }^{2}$ \\ ${ }^{1}$ CEris, ICIST, Faculdade de Ciências e Tecnologia, Universidade Nova de Lisboa, \\ Quinta da Torre, 2829-516 Caparica, Portugal \\ 2 Dipartimento di Ingegneria Civile e Ingegneria Informatica, Università di Roma \\ TorVergata, via Politecnico 1, 00133, Rome, Italy \\ E-mail: fpas@fct.unl.pt
}

\begin{abstract}
The present paper explores the capabilities of a tensegrity-inspired tower with regard to frequency tuning by shape morphing. To change the configuration of the proposed structure, shape-memory-alloy actuators are used. This actuation principle also takes advantage of the variation of the elastic modulus of shape-memory alloys associated with the martensitic transformation. The temperature modulation of the shape-memory-alloy wires is successfully achieved by Joule heating, through a proportional-integral-derivative controller, to change between a low-temperature shape and a high-temperature shape. The implementation of a short-time-Fourier-transform control algorithm allows for the correct identification of the dominant input frequency, associated with the dynamic excitation. This information is used to automatically change the configuration of the structure in order to shift its natural frequency away from that of the dynamic excitation. With this frequency tuning, one obtains a reduction of the accelerations throughout the structure up to about $80 \%$. The good performance of the proposed control approach gives promising indications regarding the use of tensegrity systems, in combination with shape-memory alloys, for shapemorphing applications, and, in particular, for self-tuning structures.
\end{abstract}

Keywords: shape-morphing, shape-memory alloys, adaptive vibration control, frequency tuning, tensegrity structures

Submitted to: Smart Mater. Struct.

\section{Introduction}

Tensegrity structures (TSs) are three-dimensional reticulated systems composed of cables and struts whose stability is provided by a self-stress state between tensioned and 
compressed members $[1,2]$. TSs have peculiar properties which make them well suited for shape-morphing applications [3-6]. Among these properties, we find the following ones. TSs are easy to fold and deploy, since a cable can go slack and occupy a small volume, and TSs are usually composed of more cables than bars. TSs can also have bars not connected to each other. TSs can be more accurately modelled, since each member of a TS is subjected to an axial force only, without bending or torsion. TSs usually require small control energy to change their shape $[7,8]$. Moreover, some TSs can present a nonlinear dynamic response originating from their special three-dimensional configuration $[9,10]$. All these properties are independent of the scale of the structure. In previous studies, TSs have been considered for foldable/deployable systems which can be compactly stowed and reliably deployed [11-17], or for smart and adaptive robotic structures whose members can act as sensors and/or actuators [18-24]. The most recent applications include a robot for rough terrain locomotion [25] and an unfurlable reflector for in-orbit telecommunication satellites [26].

An adequate choice of actuators is crucial in the development of TSs as shape-morphing structures. Here we focus on combining TSs with shape-memory-alloy (SMA) wires. On one hand, SMA wires can be a convenient actuation solution for TSs, mainly because the heavy components of the actuation system (the power unit for Joule heating in this case) can be located at anchorage points, far away from where the motion occurs. This is clearly a better solution than having heavy motorized components positioned close to or on the moving parts, or having motors remotely located by using continuous cables running over pulleys [27], which would give a more complex mechanical behavior due to friction [28]. Notice that another actuating solution previously adopted is the one provided by pneumatic muscles [29]. Although SMA actuators have limitations too, they can provide a reliable solution in many circumstances. On the other hand, TSs are the natural choice when one wants to exploit the properties of SMA in threedimensional structures. This is because of the following reasons. First, owing to the maturity of SMA wire technology, wires can be manufactured with the desired material properties. Second, the thermo-mechanical behavior of these one-dimensional elements can be predicted with a better degree of accuracy, with respect to two- or three-dimensional SMA elements. Third, since SMA wires operate in tension, they necessarily have to be embedded in stable prestressed systems, and TSs constitute the simplest instance of such systems.

In this study, a particular application of SMA-equipped TSs to vibration control is presented. This is the first step of a more general investigation that we are undertaking on these kind of structures. Usually, with an adequate placement of the compressive members, the strength-to-weight ratio of TSs is maximized for obtaining higher structural efficiencies. However, due to their inherent flexibility, relatively low structural damping, and low self-weight, tensegrity structures can experience high vibration levels in response to dynamic loadings, especially in resonance situations [30]. Several vibration control strategies have been proposed, mainly based on the modification of the structural self-stress, through small movements of active struts or ties [2,31-33]. Here, 
the frequency tuning capabilities of a tensegrity inspired structure are explored, using SMA NiTi wires as actuators. SMAs have been widely used to mitigate vibrations, both in passive control systems and adaptive control systems, taking advantage of the superelastic effect, in the former case [34-38], and of the shape-memory effect, in the latter case [39-44].

In the proposed study, the adaptive control system provides the structure with a shape-morphing ability to reduce the maximum acceleration measured under timedependent external loads [31]. The frequency tuning of the prototype is achieved by a time-frequency control algorithm, based on the short-time-Fourier transform (STFT), used to characterize the dominant excitation frequency. In order to shift the natural frequency away from the dominant frequency of the dynamic excitation, the structure can automatically change its configuration using temperature modulation of the SMA wires. The temperature control algorithm is based in a proportional-integral-derivative (PID) control approach, using Joule heating to heat the SMA wires, that is, heating the actuators using electric current.

The paper is organized as follows. In Section 2, the basic principles governing the design of shape-morphing TSs are first presented and then applied to the case of the modular tower of this study. In Section 3.1, the design of the tower and the concept of vibration control through shape morphing are detailed. In Section 3.2, the experimental setup is described. Then we present the experimental assessment of the effect of temperature modulation on the tower geometry (Section 3.3), and of the efficiency of shape morphing relative to frequency tuning (Section 3.4). In Section 4 the STFT and PID control algorithms adopted in this study are described. The results on vibration reduction performances are presented and discussed in Section 5, while our conclusions are drawn in Section 6.

\section{Basic design of shape-morphing TSs}

\subsection{Self-stress states and internal mechanisms}

TSs have been given various definitions. The most general, chosen by mathematicians, and the one used here is: prestressed pin-connected frameworks composed of bars and cables. Bars can carry either tension or compression, while cables can carry only tension. We also use the term strut to indicate a bar in compression.

A TS, or tensegrity framework, is determined by a set of $n$ points, called nodes, in the three-dimensional Euclidean space, together with a set of e edges connecting pairs of nodes, each labeled either bar or cable. If there are bars only, then we have a bar framework. We will say that $i j$ is the edge connecting nodes $i$ and $j$. Let $\mathbf{p}_{i}$ be the position vector of node $i$ with respect to a fixed reference frame. The configuration of a TS is given by the $3 n$-dimensional vector $\mathbf{p}$ which groups together all the nodal position vectors. Similarly, we can associate to each node an external force vector and a displacement vector, so that $\mathbf{f}$ and $\mathbf{u}$ are the $3 n$-dimensional vectors containing all 
nodal external forces and nodal displacements respectively. The edge $i j$ has length $l_{i j}$, and carries the internal axial force, or generalized stress, $t_{i j}$, while the edge's percent elongation is $d_{i j}$. The corresponding $e$-dimensional vectors for the whole system are $\mathbf{l}$, $\mathbf{t}$, and $\mathbf{d}$, respectively.

In the linear theory of bar frameworks [45], the equilibrium operator, A, a function of $\mathbf{p}$ only, provides the linear relation between internal forces and external forces,

$$
\mathbf{A t}=\mathbf{f},
$$

while its transpose, the kinematic compatibility operator, $\mathbf{A}^{T}$, links "small" displacements to "small" elongations,

$$
\mathbf{A}^{T} \mathbf{u}=\mathbf{d} .
$$

The elements of the nullspace of $\mathbf{A}$ are the self-stress states, $\mathbf{t}_{s}$, which are balanced by null external forces; the elements of the nullspace of $\mathbf{A}^{T}$ are the mechanisms, $\mathbf{u}_{m}$, nodal displacements which do not change the length of the edges:

$$
\mathbf{A t}_{s}=\mathbf{0}, \quad \mathbf{A}^{T} \mathbf{u}_{m}=\mathbf{0} .
$$

We say that a mechanism is nontrivial if it does not correspond to a rigid-body motion of the framework.

\subsection{Structural classification}

We denote by $s$ and $m$, respectively, the number of independent states of self-stress and the number of independent nontrivial mechanisms. Tensegrity frameworks and bar frameworks can be classified into the following four classes according to the values of $s$ and $m$ :

(i) $m=s=0$ isostatic systems;

(ii) $m>0, s=0$ underconstrained systems;

(iii) $m=0, s>0$ overconstrained systems;

(iv) $m>0, s>0$ simultaneously under- and overconstrained systems.

Clearly, $s>0$ for TSs, so that they must belong to classes (iii) and (iv). Methods for determining the class of TSs, and to check their stability, have been given in the literature, for example, in [46]. TSs in class (iii) need to satisfy a simple compatibility criterion on the sign of the self-stress: for a system to be stable there must exist a self-stress where the sign of the stress in each edge matches the corresponding label (positive for cables and positive, negative, or null for bars). TSs of interest in class (iv) are sometimes termed first-order infinitesimal mechanism, meaning that prestress imparts them a first-order geometric stiffness. The configuration of these systems is characterized by the fact that the rank of the equilibrium matrix is not maximal. Owing to the presence of mechanisms, TSs in class (iv) are usually more flexible then those in class (iii). The natural frequencies associated to mechanisms scale with the square of the prestress level, and this feature can be used for frequency tuning of the more flexible 
TSs in class (iv). On the other hand, the natural frequencies which are not associated to mechanisms are practically unaffected by changes in the prestress level $[47,48]$. We consider in this study the stiffer TSs in class (iii) for the following reason: since they have no mechanism, their dynamic response is not sensitive to changes in the prestress level, changes which can be caused by Joule-heating-induced phase transformations in SMA wires.

\subsection{Compatibility conditions}

Useful information can be obtained from the equilibrium operator. First of all, since vectors in the image of $\mathbf{A}^{T}$ are orthogonal to vectors in the nullspace of $\mathbf{A}$, we have that the following condition need to hold between kinematically admissible elongations and self-stress states:

$$
\mathbf{t}_{s} \cdot \mathbf{d}=0, \quad \mathbf{t}_{s} \in \operatorname{Ker} \mathbf{A} .
$$

Thus edges' elongations cannot be arbitrarily chosen: they are restricted by $s=$ $\operatorname{dim}(\operatorname{Ker} \mathbf{A})$ independent compatibility conditions, which means that, to change the shape of a TS, up to $s+1$ edges need to change length.

By considering a TS with $s=1$, when two edges (with non-null stress) change length, we have:

$$
t_{1} d_{1}+t_{2} d_{2}=0
$$

with the subscripts 1 and 2 referring to the two edges. The relation above shows that when these two edges are either two cables or two struts, one shorten and the other one lengthen; if the two edges are a cable and a strut, then they both shorten, or lengthen. Moreover, if the self-stress in one edge tends to zero the elongation of the other one tends to zero as well.

It is worth recalling that there are two other types of compatibility conditions a TS need to satisfy [49], these are

$$
\dot{\mathbf{t}}_{s} \cdot \mathbf{l}=0 \text {, }
$$

and

$$
\mathbf{t}_{s} \cdot \mathbf{d}=0
$$

The former condition can be interpreted as follows. Considering a TS whose stress state changes in only two edges, if they are both cables, or struts, the stress increases in one edge and decreases in the other one; if they are a cable and a strut, the stress in both edges increases, or decreases, in absolute value. The latter condition means that in a TS with many cables and few struts, the stress in struts is on average higher than that in cables, indicating that cables requires on average less power for actuation.

We now describe how condition (2) is applied to the rectangular-shaped 2D module in Fig. 1, called C2T4 module in [50]. This module is composed of two horizontal cables and two vertical cables, forming the sides of the rectangle, and by two struts, forming the diagonals. This module has one state of self-stress, $s=1$, nonnull in 
every edges, and no mechanisms in 2D, thus it belongs to class (iii). The symmetric shape change shown in Fig. 1 can be obtained by shortening the vertical cables of a predetermined amount, and by letting the horizontal cables increase in length according to (2), while keeping the length of struts unchanged. In a modular tower composed of several modules (Fig. 2), where the top horizontal cable of one module coincide with the bottom horizontal cable of the next module, there is a number of self-stress states equal to the number of modules. Notice that connecting two module in general can cause additional self-stress states to be generated. Since each self-stress state is localized at the corresponding module, condition (2) can have various implications. For example, to change the length of a horizontal cable shared between two modules, the length of two more edges, one in each of these modules, need to adjust accordingly. This is because the two self-stress states of these modules are affected. If instead it is the length of a strut or a vertical cable to change, then only one other strut or vertical cable of the same module need to adjust accordingly. This is because only the self-stress of that module is involved. It is easy to see that condition (2) is satisfied when the symmetric shape change we described above is applied simultaneously, and in equal measure, to all modules in a tower.

\section{Adaptive shape-morphing structure}

\subsection{Definition of the tower structure}

The proposed adaptive structure is inspired by the C2T4 module (Figure 1(a)). By controlling the lengths of the vertical tendons it is possible to change the overall shape of the module. The module is simply supported and has a horizontal sliding bearing to accommodate the horizontal extension $\left(u_{h}\right)$ associated with the shape-morphing, as shown in Figure 1(b). With the shape morphing, the angle $\theta_{i}$ changes to $\theta_{f}$, causing a shift on the natural frequency of the module. A parametric study comprising several finite-element (FE) models, using SAP2000, was performed in order to assess the influence of shape-morphing on the efficiency of the proposed control approach. This efficiency can be characterized by $r=f_{f} / f_{i}$, being $f_{i}$ the initial frequency of the structure and $f_{f}$ the frequency after the shape morphing. The analyzed models were twodimensional towers composed of three superposed $\mathrm{C} 2 \mathrm{~T} 4$ modules, with configurations ranging from $\theta_{i}=45 \mathrm{deg}$. to $\theta_{i}=75 \mathrm{deg}$., and a width of $145 \mathrm{~mm}$. In this parametric study, the struts were considered to be made out of pine wood ( $\left.\mathrm{E}_{w}=12 \mathrm{GPa}\right)$, with a rectangular cross section of $20 \times 10 \mathrm{~mm}^{2}$. The system is activated by a set of vertical SMA wires, providing a maximum change in length $\left(u_{v}\right)$ of about $4 \%$. The SMA tendons were considered to have a circular cross section of diameter $0.5 \mathrm{~mm}$. Their modulus of elasticity varyes between $40 \mathrm{GPa}$ and $80 \mathrm{GPa}$, corresponding to the martensitic and austenitic phases, respectively. In Figure 2, the modal configurations associated with the first vibration modes are presented, before and after shape morphing. In Figures 2 (a) and (b) $\theta_{i}=45$ deg., and in Figures 2 (c) and (d) $\theta_{i}=75$ deg.. The results of the 


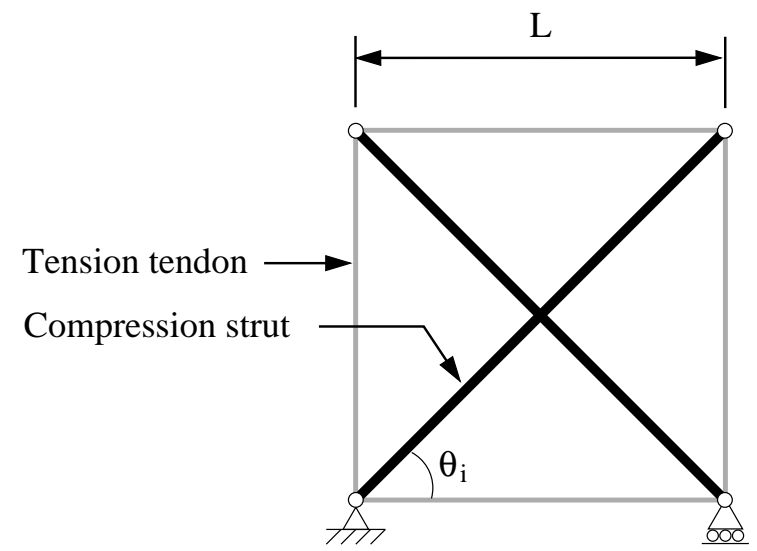

(a) Base module

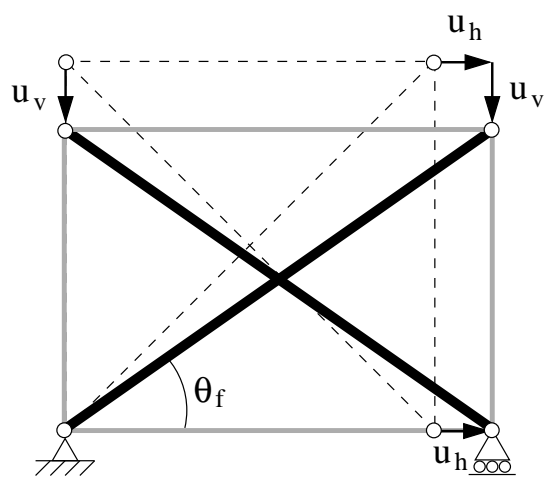

(b) Shape morphing

Figure 1: Characterization of the proposed C2T4 adaptive structure.

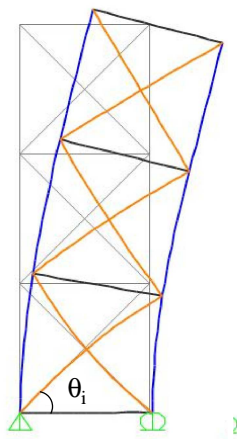

(a) Initial shape $\theta_{\mathrm{i}}=45 \mathrm{deg}$.

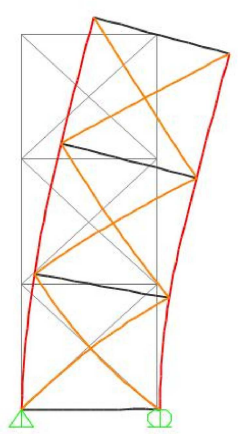

(b) Final shape $\theta_{\mathrm{i}}=45 \mathrm{deg}$.
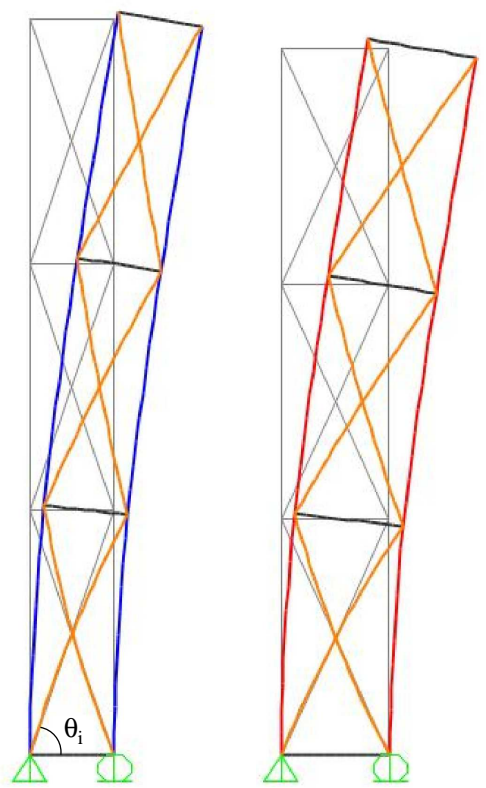

(c) Initial shape $\theta_{\mathrm{i}}=75 \mathrm{deg}$. (d) Final shape $\theta_{\mathrm{i}}=75 \mathrm{deg}$.

Figure 2: FE models for the $\theta_{i}=45 \mathrm{deg}$. and $\theta_{i}=75 \mathrm{deg}$. configurations.

parametric study in terms of the varying frequency during the shape-morphing process are presented in Figure 3, with $\theta_{i}$ ranging between $45 \mathrm{deg}$. and $75 \mathrm{deg}$. One can see 


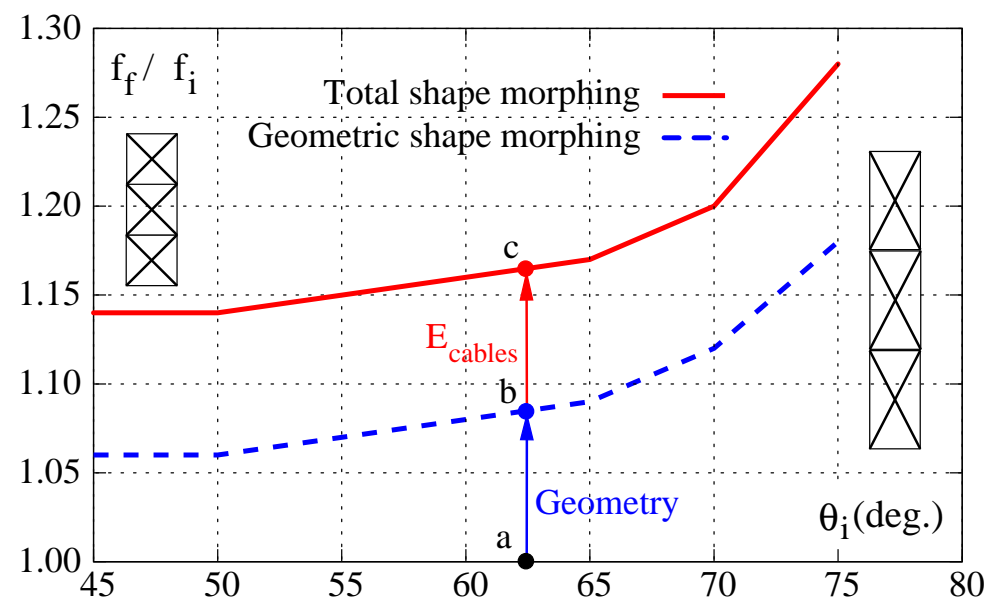

Figure 3: Frequency variation vs. strut initial angle.

that the frequency shift of the structure, during the shape-morphing process, increases with $\theta_{i}$, ranging from $14 \%$, for a $\theta_{i}$ of $45 \mathrm{deg}$., up to about $28 \%$, for a $\theta_{i}$ of $75 \mathrm{deg}$..

The frequency shift in the analyzed structures is caused by two different effects: geometric effects, directly related to the shape-morphing process itself, and physical effects related to variations on the modulus of elasticity of the SMA wires due to the thermal induced martensitic transformation. In fact, during the activation of the SMAs wires, which enables the desired change in the geometry of the tower, their modulus of elasticity also increases significantly. In Figure 3 these two components are clearly identified for the shape-morphing process of a generic structure, represented by the path $a-b-c$, in which $a-b$ represents the frequency shift associated with the geometry variation alone, and b-c, the frequency shift due to the stiffening of the SMA wires.

Taking into consideration the results yielded by the parametric study, a threedimensional tower was conceived, with a total height of $1278 \mathrm{~mm}$, comprising three $426 \times 145 \mathrm{~mm}$ modules $\left(\theta_{i}=72\right.$ deg.), as shown in Figure $4(\mathrm{a})$ (see Fig. 5 for a 3D view). This configuration corresponds to the low temperature shape (LTS), associated with the martensitic phase of the SMA tendons. In the high temperature shape (HTS), considering a $4 \%$ strain on the SMA actuators, the structure presents a total height of $1227 \mathrm{~mm}$, and a width of $188 \mathrm{~mm}$, as shown in Figure 4(b). The HTS corresponds to the austenitic phase of the SMA tendons. In order to guarantee the robustness associated with the pretension principle for tensegrity structures, the horizontal tendons were realized by extension springs with a rest length of $130 \mathrm{~mm}$ and a spring constant of $90 \mathrm{~N} / \mathrm{m}$. By introducing an initial deformation in these springs one is able to convey a self-stressed state to the LTS. 


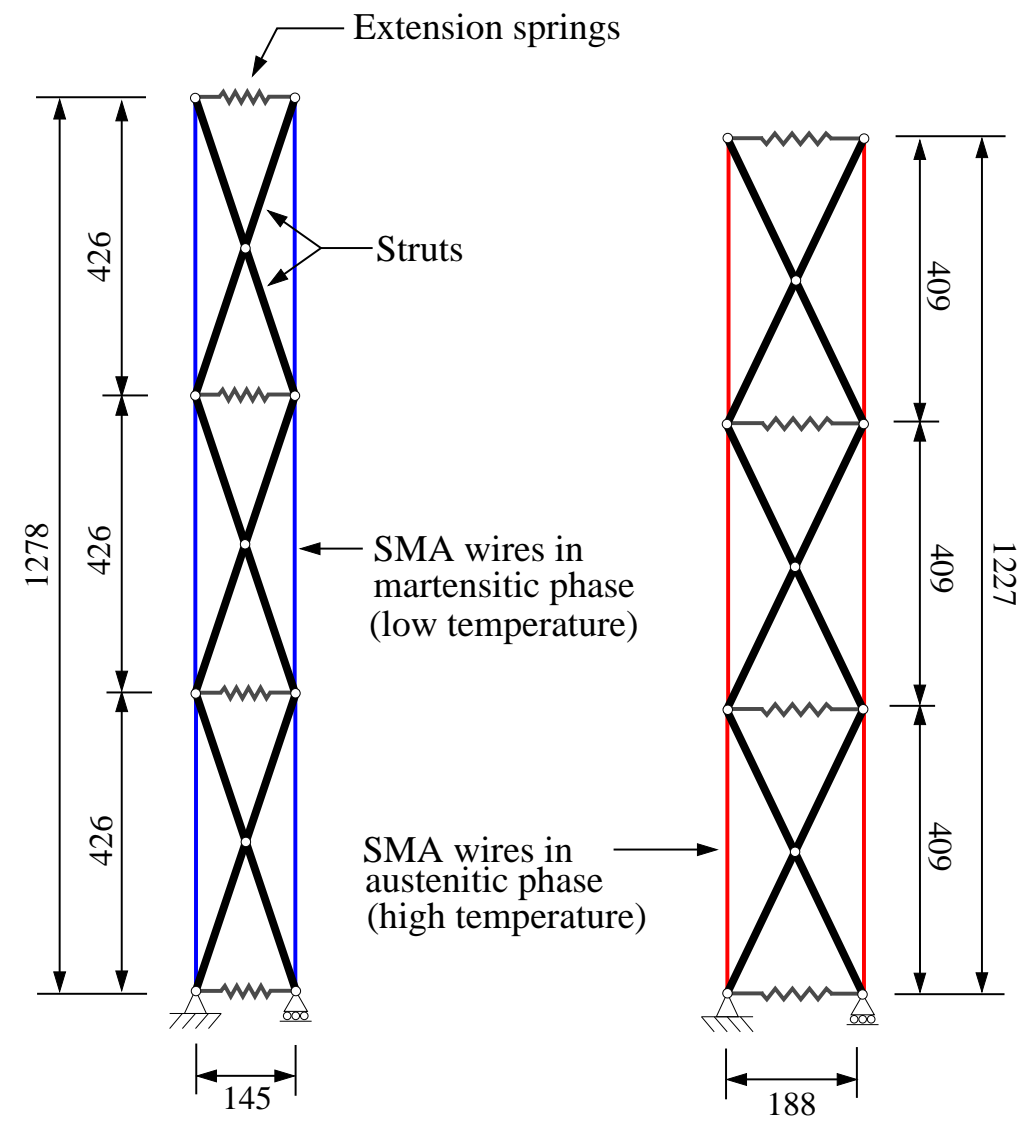

(a) High tower

(b) Short tower

Figure 4: Shape morphing between the LTS and the HTS (all dimensions in $m m$ ).

\subsection{Experimental prototype}

A general view of the prototype is shown in Figure 5, where one can see the transversal threaded stainless steel bars connecting the joints, with a total length of $200 \mathrm{~mm}$ and a diameter of $5 \mathrm{~mm}$. The prototype was mounted on a shake table (Quanser Shake Table II), in order to excite the structure. It was equipped with a set of four PCB Piezotronics, Inc. accelerometers, model 33B42, connected to a NI SCXI-1530 4 channel accelerometer amplifier. The accelerometers were placed on the three storeys of the prototype, and one was connected to the shake table. A Sorensen programmable DC power supply (PPS), model XHR 40-25, was used to allow the Joule heating of the SMA NiTi Flexinol@ Actuator Wires, provided by DYNALLOY, Inc.. The control of the SMA actuators was performed in a voltage control mode, with a maximum input current of $2 \mathrm{~A}$. The temperature of the NiTi wires was monitored by two T-type thermocouples (CopperConstantan), with a temperature reading range of $-40^{\circ} \mathrm{C}$ to $100^{\circ} \mathrm{C}$, connected to a NI SCXI-1112 8 channel thermocouple amplifier. The horizontal displacement of the sliding bearing of the prototype was monitored by a Baumer distance laser sensor, model OADM 
20I4560-S14C, connected to a NI SCXI-1520 8-Channel Universal Strain Bridge with a NI SCXI-1314 Front Terminal. The general platform for the data acquisition and control was a NI PXI-1052. A DAQ assistant express VI, using NI-DAQmx software, was used to create, edit and run the analog inputs corresponding to the voltage measurement tasks. Using an averaging process, a sample compression of the data points was performed in order to attenuate the noise derived from the readings.

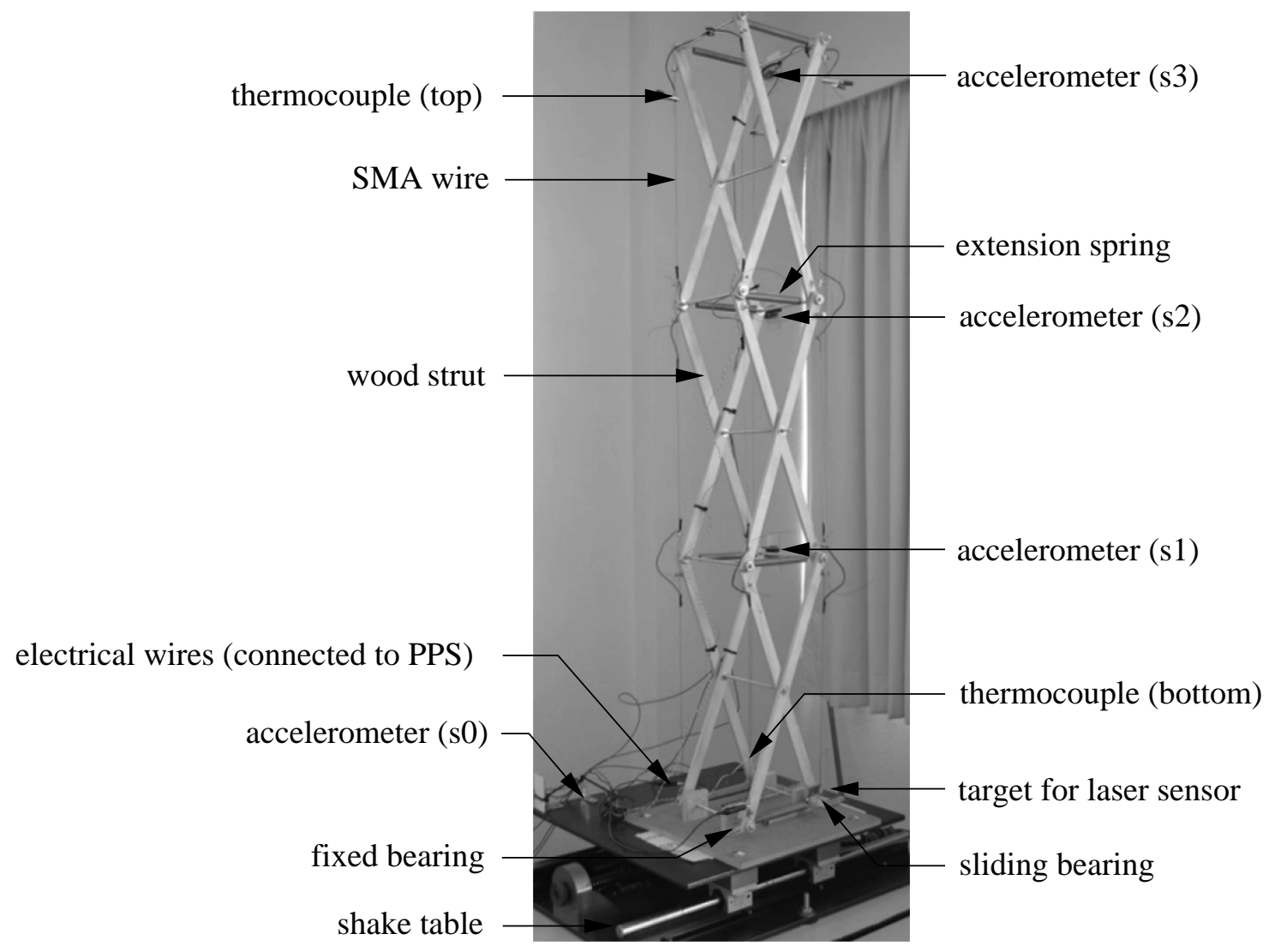

Figure 5: General view of the experimental prototype.

\subsection{Temperature modulation}

In order to characterize the phase transformation temperatures of the SMA NiTi wires, a differential scanning calorimetry (DSC) test was performed, using a SETARAM-DSC92 thermal analyzer. The temperature program comprised a thermal cycle where the sample, tested as-received, was heated up to $130^{\circ} \mathrm{C}$, held at this temperature for $6 \mathrm{~min}$, and then cooled to $-20^{\circ} \mathrm{C}$, with heating and cooling rates of $7.5^{\circ} \mathrm{C} / \mathrm{min}$. Prior to the DSC experiment, the sample was submitted to a chemical etching $(10$ vol. $\% \mathrm{HF}+45$ vol. $\% \mathrm{HNO}_{3}+45$ vol. $\left.\% \mathrm{H}_{2} \mathrm{O}\right)$ in order to remove the oxide and the layer formed by the cutting operation. The results of the DSC test are presented in Figure 6(a). One sees that the transformation from martensite to austenite, during heating, occurs within a 
temperature range between $40^{\circ} \mathrm{C}$ and $90^{\circ} \mathrm{C}$, approximately. The DSC results also show two peaks during cooling, which are associated with the presence of an intermediate R-phase, between the transformation of austenite (B2) to martensite (B19'), i.e, the transformation kinetics is $\mathrm{B} 2 \rightarrow \mathrm{R} \rightarrow \mathrm{B} 19^{\prime}$. According to these results the $\mathrm{T}_{L T S}$ was set to $20^{\circ} \mathrm{C}$ and $\mathrm{T}_{H T S}$ was set to $80^{\circ} \mathrm{C}$. To assess the efficiency of temperature modulation on the shape morphing of the prototype, the SMA wires were subjected to a thermal cycle, with temperatures ranging from $20^{\circ} \mathrm{C}$ to $80^{\circ} \mathrm{C}$. The obtained temperature vs. horizontal extension curve is plotted in Figure 6(b).

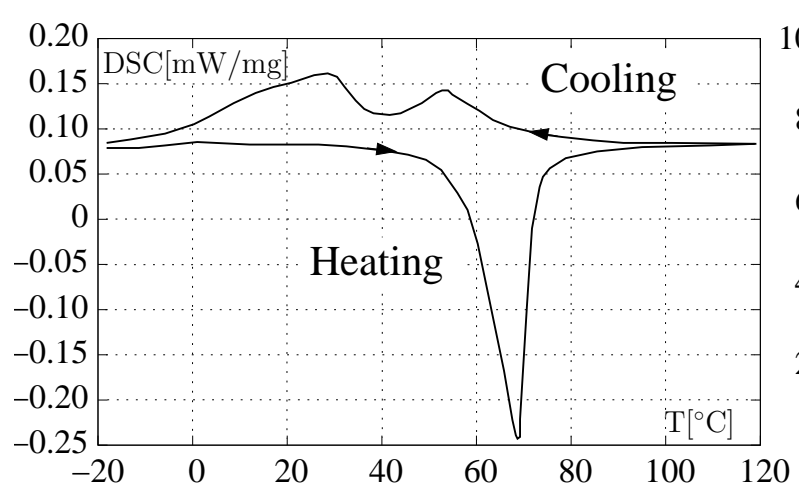

(a) DSC analysis.

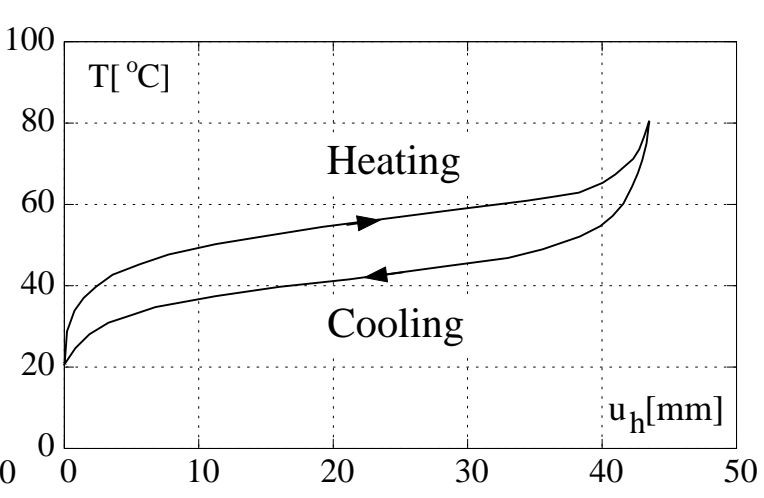

(b) Temperature vs. horizontal extension

Figure 6: Characteriation of the phase transformation temperatures.

One observes that, when passing from $20^{\circ} \mathrm{C}$ to $80^{\circ} \mathrm{C}$, the horizontal extension of the prototype amounts to $43 \mathrm{~mm}$, which amounts to a $30 \%$ variation from its original width. The thermal cycle is characterized by a hysteresis, which leads to a temperature differential between heating and cooling of about $18^{\circ} \mathrm{C}$.

\subsection{Frequency tuning}

To investigate the efficiency of the shape morphing on the frequency tuning, the prototype, was subjected to a sweep signal, with frequencies ranging from $2.0 \mathrm{~Hz}$ to 6.0 $\mathrm{Hz}$, for a set of four different temperatures $\left(20^{\circ} \mathrm{C}, 40^{\circ} \mathrm{C}, 60^{\circ} \mathrm{C}\right.$ and $\left.80^{\circ} \mathrm{C}\right)$. The response of the system, in terms of the normalized acceleration of the third storey (a/a_max) is presented in Figure 7. The results obtained with the sweep analysis show that it is possible to tune the frequency of the prototype through shape-morphing. In fact, by changing the configuration of the prototype between the LTS and HTS, one is able to shift its natural frequency from $4.3 \mathrm{~Hz}$ to $5.2 \mathrm{~Hz}$. This represents a $21 \%$ variation of the original frequency, which is in close agreement with the numerical results presented in Figure 3. During this shape morphing, damping slightly decreases from $6.2 \%$ to $5.2 \%$. For the $40^{\circ} \mathrm{C}$, the frequency remains mostly unaltered. At $\mathrm{T}=60^{\circ} \mathrm{C}$, there is a frequency shift of about $10 \%$. Once the acceleration amplification curves of the prototype, associated with the LTS and the HTS, have been characterized, is possible to establish which is the most suitable configuration for a given input frequency, i.e. 


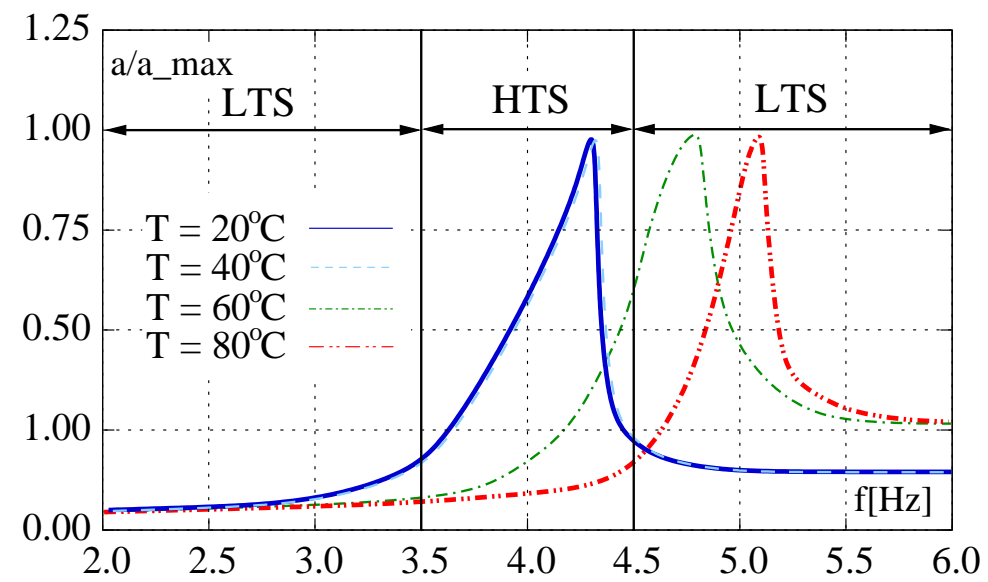

Figure 7: Frequency sweep between 2.0 and $6.0 \mathrm{~Hz}$.

the configuration that minimizes the system's response in terms of acceleration. Three frequency input windows were considered, as shown in Figure 7: below $3.5 \mathrm{~Hz}$, above $4.5 \mathrm{~Hz}$, and in-between these values. For input frequencies below $3.5 \mathrm{~Hz}$ and above $4.5 \mathrm{~Hz}$, the adequate configuration of the prototype would be the LTS, since in these frequency input ranges it is this configuration that minimizes the system's response. For input frequencies between $3.5 \mathrm{~Hz}$ and $4.5 \mathrm{~Hz}$, the adequate configuration would be the HTS, for the same reason.

\section{Control algorithms}

In this section, a brief description of the control algorithms that confer the tower its frequency self-tuning capabilities is presented. The first algorithm is based on the STFT and enables the tower to identify the dominant excitation frequency and to define the adequate configuration according to the frequency input windows presented in Figure 7. The second algorithm is based in a proportional-integral-derivative (PID) control approach, which has been found to give good results for temperature modulation in SMAs using Joule heating [51].

\subsection{STFT control algorithm}

In the STFT control algorithm, the time-domain input signal is first partitioned into several disjointed or overlapped blocks, with a certain buffer length, which is a region of a physical memory storage used to temporarily store data. Multiplying the signal with a window function, which is nonzero for only a short period of time, improves the spectral characteristics of the signal. The discrete Fourier transform is then applied to each block, after extending the signal with zeros (zero padding), to make the signal have a power-of-two samples, which makes the algorithm more efficient. Because each block occupies different time periods, the resulting STFT indicates the spectral content 
of the signal at each corresponding time period. By moving the sliding window, one obtains the spectral content of the signal over different time intervals. Therefore, the STFT is a function of time and frequency that indicates how the spectral content of a signal evolves over time. More details about the mathematical implementation of the STFT algorithm can be found in [52]. The natural frequency of the prototype is continuously adjusted through shape morphing by the STFT control algorithm. The current dominant frequency of response is identified according to the STFT algorithm shown in Figure 8. The top storey acceleration is the only feedback used in the STFT algorithm. The frequency of the prototype is tuned in order to minimize the dynamic response, according to the frequency input windows previously defined.

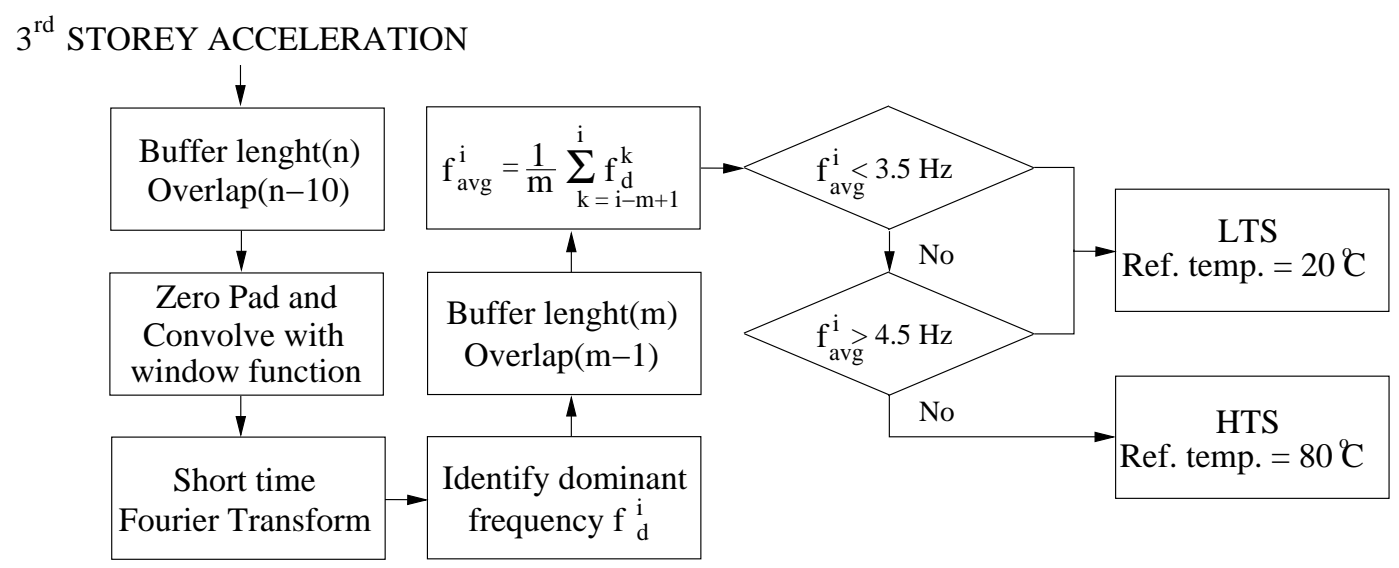

Figure 8: STFT control algorithm.

\subsection{PID control algorithm}

As mentioned ealier, the frequency tuning of the prototype is achieved by temperature modulation of the SMA wires. The reference temperature for a given instant is defined by the STFT control algorithm. This temperature is the input reference for the temperature control algorithm, which is based in a PID control approach (represented in Figure 9). Being a closed-loop control system, the temperature output signal is fed back to the summing point and compared with the reference temperature input, yielding the actuating error signal. The output signal of the controller is the analog voltage reference input for the PPS. The feedback-path transfer function corresponds to the thermocouple in the SMA wire, which measures the output variable in order to make it comparable with the reference input signal, resulting in the feedback temperature signal.

\section{Results and discussion}

The STFT spectrogram, which is a visual representation of the spectrum of frequencies as they vary with time (for a frequency input variation between $2.0 \mathrm{~Hz}$ and $8.0 \mathrm{~Hz}$ ), is 


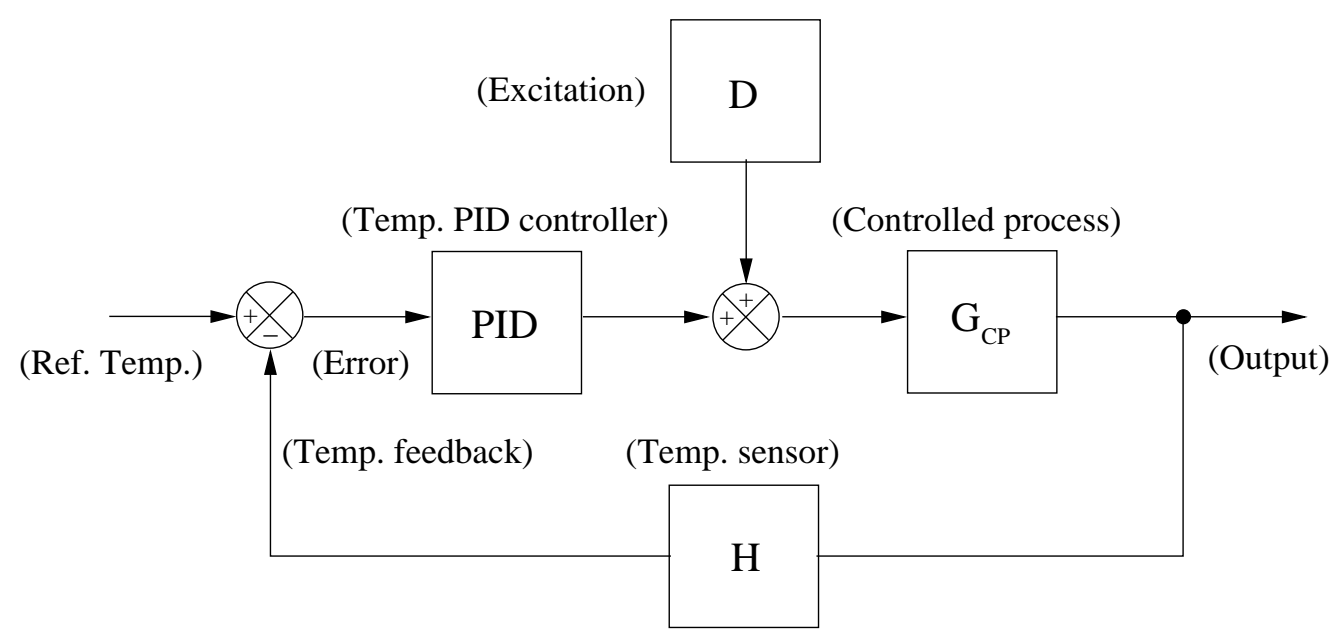

Figure 9: Temperature control algorithm.

shown in Figure 10. The input frequency variation is overlaid on the spectrogram to show its correlation with the dominant frequency identified by STFT algorithm. It is clear that the STFT algorithm identifies the dominant input frequency.

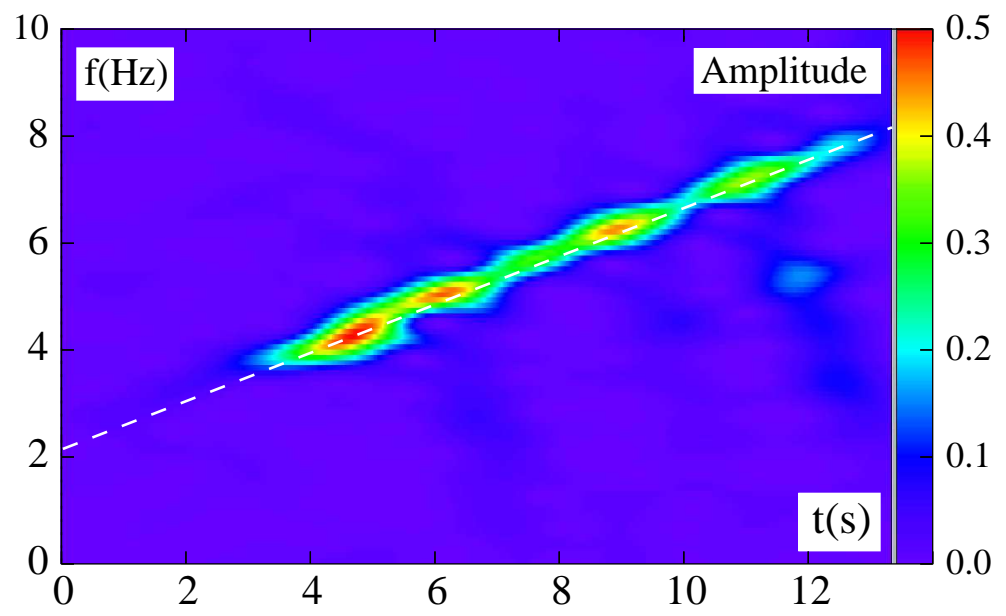

Figure 10: Time-frequency distribution overlaid with frequency input variation, between $2.0 \mathrm{~Hz}$ and $8.0 \mathrm{~Hz}$.

The thermo-mechanical behavior of the prototype during shape morphing is presented in Figures 11(a) and (b). One can see that the response of the prototype during heating is much faster than during cooling. This is due to the fact that heating is controlled by the Joule heating and cooling mostly depends on the convection mechanisms between the SMA wires and the surrounding air. The presence of the intermediate R-phase doesn't seem to hinder the performance of the proposed temperature control algorithm.

The evaluation of the performance is based on the ability of the control system to 


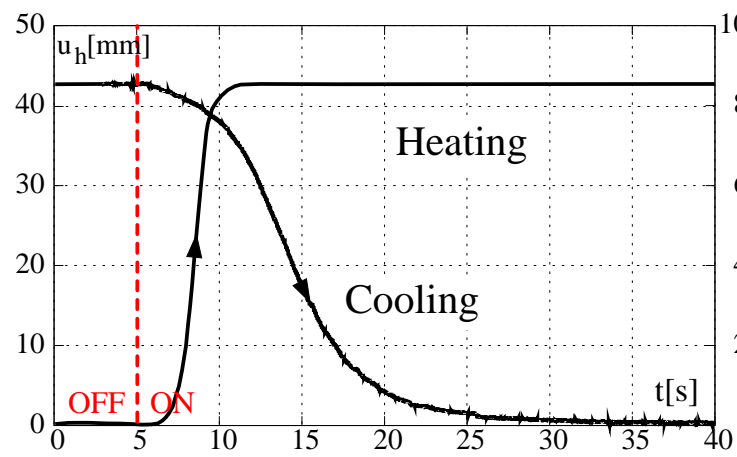

(a) Horizontal extension vs. time.

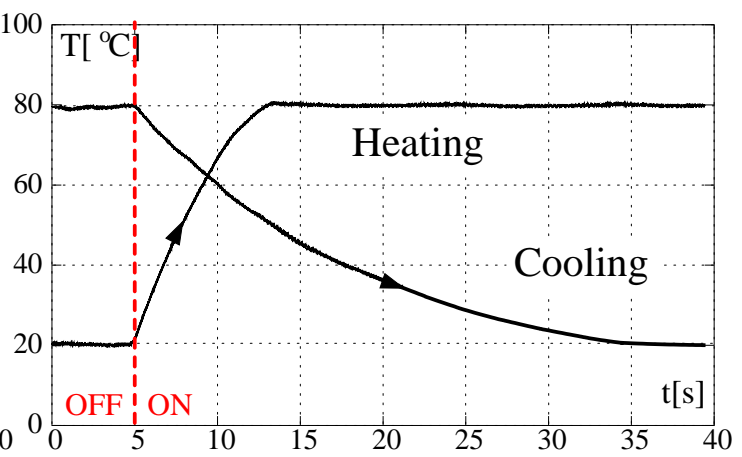

(b) Temperature. vs. time.

Figure 11: Horizontal extension and temperature vs. time during shape morphing.

reduce the root mean square (RMS) and the absolute accelerations of the prototype, associated with shape morphing. In Figures $12(\mathrm{a})$ and (b) one can see the reduction of the peak and RMS accelerations, respectively, in the third storey, when shape morphing from the LTS to the HTS occurs, for an input frequency of 4.3 Hz. In Figures 13(a) and (b) show the corresponding acceleration graphs, when shape morphing from the LTS to the HTS occurs, for an input frequency of $5.2 \mathrm{~Hz}$.

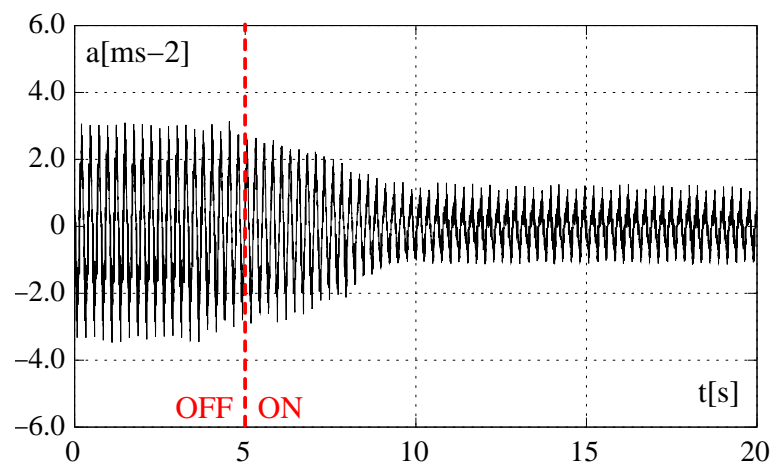

(a) Acceleration time-history (Third storey).

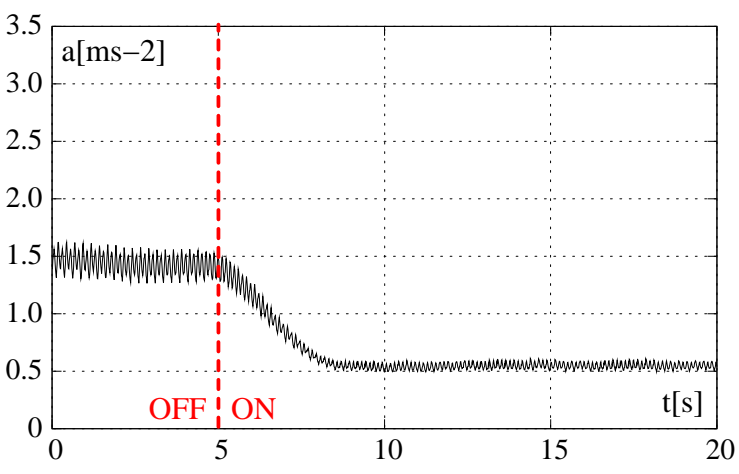

(b) Acceleration RMS time-history (Third storey).

Figure 12: Shape morphing from LTS to $\operatorname{HTS}\left(\mathrm{f}_{i}=4.3 \mathrm{~Hz}\right)$.

Detailed peak and RMS responses are presented in Tables 1 and 2, comparing the acceleration responses of the uncontrolled structure, and of the structure with shape-morphing abilities.

One can see that the performance of the proposed adaptive system is very good, with reductions of the peak and RMS accelerations up to 80\%, when shifting from HTS to LTS, and up to about $60 \%$, when shifting from LTS to HTS. 


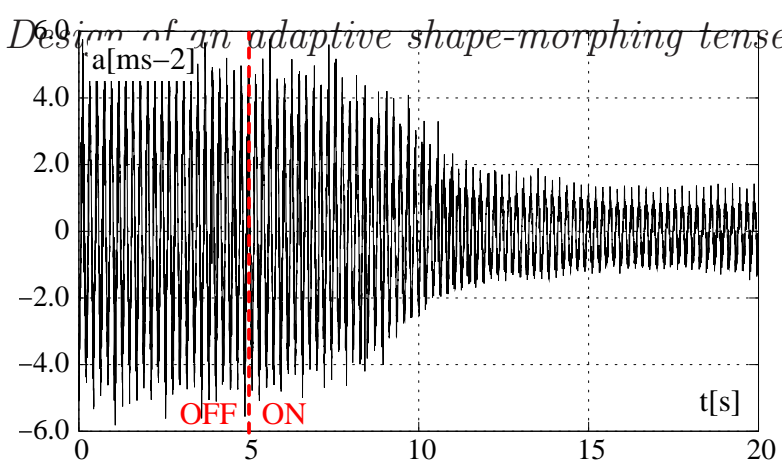

(a) Acceleration time-history (Third storey).

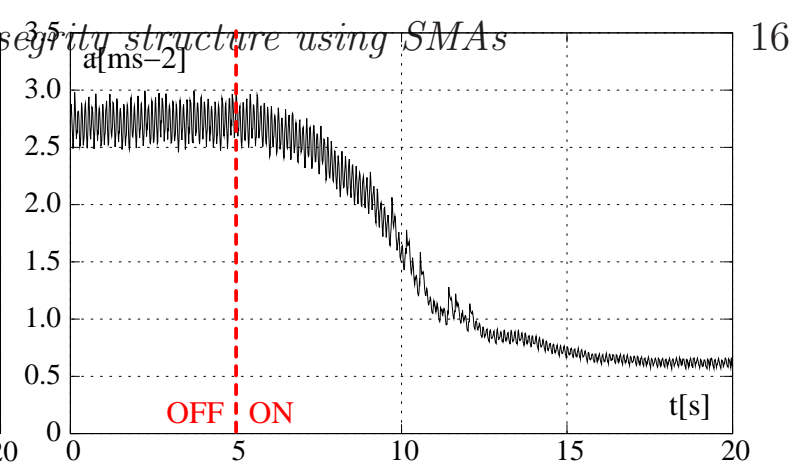

(b) Acceleration RMS time-history (Third storey).

Figure 13: Shape morphing from HTS to LTS $\left(\mathrm{f}_{i}=5.2 \mathrm{~Hz}\right)$.

Table 1: Comparison of peak and RMS acceleration responses: LTS to HTS.

\begin{tabular}{|c|c|c|c|c|c|c|}
\hline $\mathrm{f}_{i}=4.3 \mathrm{~Hz}$ & \multicolumn{3}{|c|}{ peak acceleration [ms-2] } & \multicolumn{3}{c|}{ RMS acceleration [ms-2] } \\
\hline storey number & uncontrolled & controlled & variation & uncontrolled & controlled & variation \\
\hline 1 & 1.23 & 0.68 & $-45 \%$ & 0.60 & 0.27 & $-55 \%$ \\
\hline 2 & 1.76 & 0.74 & $-58 \%$ & 1.02 & 0.37 & $-64 \%$ \\
\hline 3 & 3.05 & 1.23 & $-60 \%$ & 1.40 & 0.51 & $-64 \%$ \\
\hline
\end{tabular}

Table 2: Comparison of peak and RMS acceleration responses: HTS to LTS.

\begin{tabular}{|c|c|c|c|c|c|c|}
\hline $\mathrm{f}_{i}=5.2 \mathrm{~Hz}$ & \multicolumn{3}{|c|}{ peak acceleration } & \multicolumn{3}{c|}{ RMS acceleration } \\
\hline storey number & uncontrolled & controlled & variation & uncontrolled & controlled & variation \\
\hline 1 & 1.92 & 0.47 & $-76 \%$ & 0.94 & 0.26 & $-72 \%$ \\
\hline 2 & 3.40 & 0.63 & $-81 \%$ & 1.63 & 0.32 & $-80 \%$ \\
\hline 3 & 5.90 & 1.33 & $-78 \%$ & 2.75 & 0.69 & $-75 \%$ \\
\hline
\end{tabular}

\section{Conclusions}

The present paper addresses the dynamic response of an adaptive shape-morphing tower structure with frequency self-tuning capabilities. This study showed that tensegrity inspired structures can excel in adaptive dynamic control applications based on frequency tuning. From the performed analysis one can draw the following conclusions:

(i) The use of active SMA tendons directly embedded in the structure is a viable design solution to enable shape morphing and to reduce the peak acceleration.

(ii) By increasing the angle of the struts of the original C2T4 base module one is able to increase the frequency shift associated with shape-morphing, leading to higher frequency tuning capabilities for the system.

(iii) The frequency shift of the structure is associated with both geometric and physical effects, the latter due to variations on the elastic modulus of the SMA wires associated with the development of the martensitic transformation. This is one of the main reasons that makes SMA activation so appealing for shape-morphing 
TSs.

(iv) The proposed STFT based control algorithm is effective in identifying the dominant input frequency associated with the dynamic excitation.

(v) With the temperature modulation of the SMA wires, through Joule heating, based on a PID control algorithm, is possible to obtain an adequate response for the tower structure.

(vi) Based on the ability of the control system to successfully reduce the root mean square (RMS) and the absolute accelerations of the prototype (up to $80 \%$ ), it is possible to conclude that the proposed control approach shows great potential for vibration mitigation in engineering applications.

\section{Acknowledgments}

Cooperation with Professor F. M. Braz Fernandes is gratefully acknowledged. We also wish to thank an anonymous reviewer for several corrections and useful suggestions.

\section{References}

[1] R. Motro. Tensegrity systems: the state of the art. Journal of Space Structures, 2(7):7583, 1992.

[2] N. Bel Hadj Ali and I.F.C. Smith. Dynamic behavior and vibration control of a tensegrity structure. International Journal of Solids and Structures, 47(9):1285 - 1296, 2010.

[3] M. Defossez. Shape memory effect in tensegrity structures. Mechanics Research Communications, 30:311-316, 2003.

[4] E. Fest, K. Shea, and I. F. C. Smith. Active tensegrity structure. Journal of Structural Engineering, 130(10):1454-1465, 2004.

[5] M. Schenk, S. D. Guest, and J. L. Herder. Zero stiffness tensegrity structures. International Journal of Solids and Structures, 44(20):6569-6583, 2007.

[6] M. Arsenault and C. M. Gosselin. Kinematic and static analysis of a 3-pups spatial tensegrity mechanism. Mechanism and Machine Theory, 44(1):162-179, 2009.

[7] Sergi Hernndez Juan and Josep M. Mirats Tur. Tensegrity frameworks: Static analysis review. Mechanism and Machine Theory, 43(7):859 - 881, 2008.

[8] Cornel Sultan, Martin Corless, and Robert E. Skelton. Linear dynamics of tensegrity structures. Engineering Structures, 24(6):671 - 685, 2002.

[9] I. J. Oppenheim and W. O. Williams. Geometric effects in an elastic tensegrity structure. Journal of Elasticity, 59(1-3):51-65, 2000.

[10] I. J. Oppenheim and W. O. Williams. Vibration of an elastic tensegrity structure. European Journal of Mechanics A/ Solids, 20:1023-1031, 2001.

[11] H. Furuya. Concept of deployable tensegrity structures in space applications. International Journal of Space Structures, 7(2):143-151, 1992.

[12] A. Hanaor. Double-layer tensegrity grids as deployable structures. International Journal of Space Structures, 8(1-2):135-143, 1993.

[13] I. J. Oppenheim and W. O. Williams. Tensegrity prisms as adaptive structures. Adaptive Structures and Material Systems ASME, 54:113-120, 1997.

[14] M. Bouderbala and R. Motro. Folding tensegrity systems. In Proceedings of IUTAM/IASS Symposium on Deployable Structures: Theory and Applications, Cambridge, U.K. Proceedings of IUTAM/IASS Symposium on Deployable Structures: Theory and Applications, Cambridge, U.K., 1998. 
[15] C. Sultan and R.E. Skelton. Tendon control deployment of tensegrity structures. In Proceedings of SPIE - Volume 3323, Smart Structures and Materials 1998: Mathematics and Control in Smart Structures, Vasundara V. Varadan, Editor, July 1998, pp. 455-466, 1998.

[16] C. Crane, B. Knight, Duffy J., and J. Rooney. Innovative deployable antenna developments using tensegrity design. In Proc. 41th AIAA/ASME/ASCE/AHS/ASC Structures, Structural Dynamics, and Materials Conference, Atlanta, GA, USA, 36 April 2000, 2000.

[17] A. G. Tibert. Deployable tensegrity structures for space applications. PhD thesis, Royal Institute of Technology, Stockholm, Sweden, 2002.

[18] R. E. Skelton, J. W. Helton, R. Adhikari, J. P. Pinaud, and W. Chan. The Mechanical Systems Design Handbook: Modeling, Measurement and Control, chapter An introduction to the mechanics of tensegrity structures. CRC press, London, U.K., 2001.

[19] J. B. Aldrich, R. E. Skelton, and K. Kreutz-Delgado. Control synthesis for a class of light and agile robotic tensegrity structures. In Proceedings of the IEEE American Control Conference, Denver, Colorado. Proceedings of the IEEE American Control Conference, Denver, Colorado, 2003.

[20] C. Paul, H. Lipson, and F. J. Valero Cuevas. Design and control of tensegrity robots for locomotion. IEEE Transactions on Robotics, 22(5):944-957, 2005.

[21] A. Graells Rovira and J. M. MiratsTur. Control and simulation of a tensegrity-based mobile robot. Robotics and Autonomous Systems, 57:526-535, 2009.

[22] O. Shai, I. Tehori, A. Bronfeld, M. Slavutin, and U. Ben-Hanan. Adjustable tensegrity robot based on assur graph principle. In Proceedings of IMECE2009, 2009.

[23] M. Shibata and S. Hirai. Rolling locomotion of deformable tensegrity structure. In Proceedings of WSPC, 2009.

[24] Y. Ushigome, K. Nishimura, and Hirose M. Structured creature: a prototype of moving space structure. In Proceedings of ACE2008, Yokohama, Japan, 2008.

[25] J. Bruce, K. Caluwaerts, A. Iscen, A. P. Sabelhaus, and V. SunSpiral. Design and evolution of a modular tensegrity robot platform. In IEEE International Conference on Robotics and Automation (ICRA), 2014.

[26] V.S. Zolesi, P.L. Ganga, L. Scolamiero, A. Micheletti, P. Podio-Guidugli, A.G. Tibert, A. Donati, and M. Ghiozzi. On an innovative deployment concept for large space structures. In Proceedings of 42nd International Conference on Environmental Systems (ICES), AIAA 2012-3601, Reston Va, USA: American Institute of Aeronautics and Astronautics (AIAA), San Diego, CA, USA, 15-19 July, 2012.

[27] K. W. Moored and H. Bart-Smith. Investigation of clustered actuation in tensegrity structures. International Journal of Solids and Structures, 46:3272-3281, 2009.

[28] N. Veuve, S. Dalil Safaei, and I. F. C Smith. Deployment of a tensegrity footbridge. Journal of Structural Engineering, 04015021, 2015.

[29] S. Hirai, Y. Koizumi, and M. Shibata. Active shaping of a tensegrity robot via pre-pressure. In IEEE/ASME Int. Conf. on Advanced Intelligent Mechatronics (AIM), 2013.

[30] Nizar Bel Hadj Ali, Landolf Rhode-Barbarigos, Alberto A. Pascual Albi, and Ian F.C. Smith. Design optimization and dynamic analysis of a tensegrity-based footbridge. Engineering Structures, 32(11):3650 - 3659, 2010.

[31] Bernard Adam and Ian F.C. Smith. Active tensegrity: A control framework for an adaptive civil-engineering structure. Computers \& Structures, 86(2324):2215 - 2223, 2008.

[32] S. Kmet, P. Platko, and M. Mojdis. Analysis of adaptive light-weight structures. Procedia Engineering, 40(0):199 - 204, 2012.

[33] M Ganesh Raja and S Narayanan. Active control of tensegrity structures under random excitation. Smart Materials and Structures, 16(3):809, 2007.

[34] B. Andrawes and R. DesRoches. Comparison Between Shape Memory Alloy Restrainers and and Other Bridge Retrofit Devices. ASCE Journal of Bridge Engineering, 12(6):700-709, 2007.

[35] R. DesRoches and M. Delemont. Seismic retrofit of simply supported bridges using shape memory 
alloys. Engineering Structures, 24:325-332, 2002.

[36] J. McCormick, R. DesRoches, D. Fugazza, and F. Auricchio. Seismic Vibration Control Using Superelastic Shape Memory Alloys. Journal of Engineering Materials and Technology, 128(3):294-301, 2006.

[37] R. Johnson, J. E. Padgett, M. E. Maragakis, R. DesRoches, and M. S. Saiidi. Large scale testing of Nitinol shape-memory alloy devices for retrofitting of bridges. Smart Materials and Structures, 17(3), 2008.

[38] Jamie Ellen Padgett, Reginald DesRoches, and Russell Ehlinger. Experimental response modification of a four-span bridge retrofit with shape memory alloys. Structural Control and Health Monitoring, 17(6), 2010.

[39] A. Baz, S. Poh, J. Ro, and J. Gilheany. Control of the natural frequencies of Nitinol-reinforced composite beams. Journal of Sound and Vibration, 185(1):171-185, 1995.

[40] A. Baz, T. Chen, and J. Ro. Shape control of Nitinol-reinforced composite beams. Composites Part B: Engineering, 31(8):631-642, 2000.

[41] K. A. Williams, G. T. C. Chiu, and R. J. Bernhard. Dynamic modelling of a shape memory alloy adaptive tuned vibration absorber. Journal of Sound and Vibration, 280(1-2):211-234, 2005.

[42] M. A. Savi, A. S. de Paula, and D. C. Lagoudas. Numerical investigation of an adaptive vibration absorber using shape memory alloys. Journal of Intelligent Material Systems and Structures, 22(1):67-80, 2011.

[43] L. Wang and R. V. N. Melnik. Nonlinear dynamics of shape memory alloy oscillators in tuning structural vibration frequencies. Mechatronics, 22(8):1085-1096, 2012.

[44] R. Aguiar, M. Savi, and P. Pacheco. Experimental investigation of vibration reduction using shape memory alloys. Journal of Intelligent Material Systems and Structures, 24(2):247-261, 2012.

[45] S. Pellegrino and C. R. Calladine. Matrix analysis of statically and kinematically indeterminate frameworks. International Journal of Solids and Structures, 22:409-428, 1986.

[46] A. Micheletti and W. O. Williams. A marching procedure for form-finding for tensegrity structures. Journal of Mechanics of Materials and Structures, 2(5):857-882, 2007.

[47] H. Murakami and y. Nishimura. Initial shape finding and modal analyses of cyclic right-cylindrical tensegrity modules. Computers and Structures, 79:891-917, 2001.

[48] Nasseradeen Ashwear and Anders Eriksson. Natural frequencies describe the pre-stress in tensegrity structures. Computers \& Structures, 138(0):162 - 171, 2014.

[49] A. Micheletti. On generalized reciprocal diagrams for self-stressed frameworks. International Journal of Space Structures, 23(3):153-166, 2008.

[50] R. E. Skelton and M. C. de Oliveira. Tensegrity systems. Springer, 2009.

[51] Y. H. Teh and R. Featherstone. An architecture for fast and accurate control of shape memory alloy actuators. The International Journal of Robotics Research, 27(5):595-611, 2008.

[52] Satish Nagarajaiah and Nadathur Varadarajan. Short time fourier transform algorithm for wind response control of buildings with variable stiffness $\{$ TMD\}. Engineering Structures, 27(3):431 - 441, 2005. 